\title{
Ranking Function Application for Optimal Solution of Fractional Programming Problem
}

\author{
Rasha Jalal Mitlif* \\ 10096@uotechnology.edu.iq \\ Applied Sciences Department, Mathematics and Computer Applications, University of \\ Technology, Baghdad, Iraq.
}

\begin{abstract}
The aim of this paper is to suggest a solution procedure to fractional programming problem based on new ranking function (RF) with triangular fuzzy number (TFN) based on alpha cuts sets of fuzzy numbers. In the present procedure the linear fractional programming (LFP) problems is converted into linear programming problems. We concentrate on linear programming problem problems in which the coefficients of objective function are fuzzy numbers, the right- hand side are fuzzy numbers too, then solving these linear programming problems by using a new ranking function. The obtained linear programming problem can be solved using win QSB program (simplex method) which yields an optimal solution of the linear fractional programming problem. Illustrated examples and comparisons with previous approaches are included to evince the feasibility of the proposed approach.
\end{abstract}

Keywords: Fuzzy set, Fuzzy number, linear fractional programming, Ranking function, Triangular fuzzy number.

\footnotetext{
* Corresponding author
} 


\section{Introduction}

Ranking method is used in many optimization problems in an economic environment. In an organization various activities such as planning, execution, and other process takes place continuously. This requires careful observation of various parameters which are all in uncertain in nature due the competitive business environment globally. In decision making a ranking of fuzzy number have an important role to express fuzzy numbers in crisp value.

Many authors studied adopted ranking function to solve fractional programming problems and linear programming problems by proposed new efficient methods $[5,14,1]$.

Ranking functions are an interactive approach for solving fuzzy parameters together with many techniques $[7,9,8$, $16,22]$. They have proposed different methods for solving fuzzy linear programming problems are reduced to crisp linear programming problems and the resulted crisp linear programming problems is solved to obtain an optimal solution of the fuzzy linear programming problems $[18,17,4,3,13,20]$.

Also, many researchers presented a ranking procedure fully fuzzy numbers with linear, multi objective programming problems function can be reduced to a crisp value problem to obtain the optimal solution [10, 2, 15]. The authors Stephen and Mohamed in [6] introduced a new procedure to treat fully fuzzy integer linear programming problems with ranking function.
In the present work, a new ranking function method is suggested to treat some linear fractional programming problem. A special procedure is used in order to convert problem into a linear programming problems. After that, the presented new ranking function applied to solve the converted fuzzy linear programming problems. To illustrate the proposed method, some numerical examples are solved.

The remainder sections of this paper are marshaled as follows: In section 2, some basic definition and triangular fuzzy numbers are reviewed while section 3 proposes a new ranking function for the solution of LFPP. Section 4 explains the mathematical expression for linear fractional function. In section 5 , the proposed new technique for solving the (FLFP) problem is discussed. Numerical examples are included in section 6 to show the efficiency of the suggested method. Finally, some conclude conclusions are listed in section 7.

\section{Preliminaries}

Some useful concepts concerning the fuzzy triangular numbers are presented throughout this section.

\section{Definition 2.1 [12]}

Suppose that $\tilde{A}$ is a fuzzy set defined on the set $\mathrm{R}$ then it is said to be a fuzzy number, if $\tilde{A}: \mathrm{R} \rightarrow[0,1]$ has the following properties: 
1. $\tilde{A}\left(\lambda x_{1}+(1-\lambda) x_{2}\right) \geq \min$ $\left\{\tilde{A}\left(x_{1}\right), \tilde{A}\left(x_{2}\right)\right\}, \in[0,1]$, for all $x_{1}$, $x_{2} \in R$,

2. There exists an $\mathrm{x} \in \mathrm{R}$ such that $\widetilde{\mathrm{A}}(\mathrm{x})=1$,

3. The fuzzy set $\widetilde{A}$ is piecewise continuous.

\section{Definition 2.2 [19]}

Suppose that $\tilde{A}=(a, b, c)$ is a fuzzy number then it is called a triangular fuzzy number if the following condition is satisfied

$$
\tilde{A}(X)= \begin{cases}\frac{(x-a)}{b-a} & a \leq x \leq b \\ \frac{(c-x)}{c-b} & b \leq x \leq c \\ 0 & \text { else }\end{cases}
$$

\section{Ranking function}

A ranking function of a fuzzy number $R$ : $F(R) \rightarrow R$ is the set of all fuzzy numbers defined on $R$ : which maps each fuzzy number into a real number

\section{The New Ranking Function}

$$
\tilde{A}(X)= \begin{cases}\frac{(x-a)}{b-a} & a \leq x \leq b \\ \frac{(c-x)}{c-b} & b \leq x \leq c \\ 0 & \text { else }\end{cases}
$$

Assume $\alpha \in[0,1]$, then

$$
\begin{aligned}
\frac{(x-a)}{b-a}=\alpha & \rightarrow x=a+\alpha(b-a) \\
& =\inf \tilde{A}(\alpha) . \\
\frac{(c-x)}{c-b}=\alpha & \rightarrow x=c-\alpha(c-b) \\
& =\sup \tilde{A}(\alpha) .
\end{aligned}
$$

By the ranking function

$$
\begin{aligned}
& R(\tilde{A}) \\
& =\frac{\int_{0}^{1} \alpha^{4}[w \inf (\widetilde{A}(\alpha))+(1-w) \sup (\widetilde{A}(\alpha))] d \alpha}{\int_{0}^{1} \alpha^{4} d \alpha}
\end{aligned}
$$

$R(\tilde{A})$
$=\frac{\int_{0}^{1} \alpha^{4}[w(a+\alpha(b-a))+(1-w)(c-\alpha(c-b))] d \alpha}{\int_{0}^{1} \alpha^{4} d \alpha}$

\section{$R(\tilde{A})$}

$=\frac{\int_{0}^{1} \alpha^{4}[w a+w \alpha(b-a)+(1-w) c-(1-w) \alpha(c-b)] d \alpha}{\int_{0}^{1} \alpha^{4} d \alpha}$

$R(\tilde{A})$
$=\frac{\int_{0}^{1}\left[\alpha^{4} w a+\alpha^{5} w(b-a)+(1-w) \alpha^{4} c-(1-w) \alpha^{5} c+(1-w) \alpha^{5} b\right] d \alpha}{\int_{0}^{1} \alpha^{4} d \alpha}$

$R(\tilde{A})$

$=\frac{\left.\left[\frac{\alpha^{5}}{5} w a+\frac{\alpha^{6}}{6} w b-\frac{\alpha^{6}}{6} w a+\frac{\alpha^{5}}{5}(1-w) c-\frac{\alpha^{6}}{6}(1-w) c+\frac{\alpha^{6}}{6}(1-w) b\right]\right|_{0} ^{1}}{\left.\frac{\alpha^{5}}{5}\right|_{0} ^{1}}$

$$
\begin{gathered}
R(\tilde{A}) \\
=\frac{\frac{1}{5} w a+\frac{1}{6} w b-\frac{1}{6} w a+\frac{1}{5}(1-w) c-\frac{1}{6}(1-w) c+\frac{1}{6}(1-w) b}{\frac{1}{5}} \\
R(\tilde{A})=\frac{1}{6}[w(a-c)+5 * b+c]
\end{gathered}
$$

\section{Linear fractional programming problem [21]}

The mathematical expression for linear fractional function is given by

$\operatorname{Maximize} F(X)=\frac{c^{T} X+\gamma}{d^{T} X+\beta}$

Subject to,

$b\}, x \geq 0$.

$$
x \in X=\{x: A x \leq
$$

where $\quad c, d$ and $x \in \mathbb{R}^{n}, A$ is an $(m+$ $n) n$ matrix,$b \in \mathbb{R}^{m+n}$ and $\gamma$ and $\beta$ are scalars. 
Note that the nonnegative conditions are included in the set of constraints as well as the condition $d^{T} X+\beta>0$ is satisfied over the set $X$.

In order to convert the original LFP problem, a feasible point $x^{*}$ of the set $X i$ s chosen to get the following vector at a certain feasible point $x^{*}$

$F^{*}=F\left(x^{*}\right)=\frac{c^{T} x^{*}+\gamma}{d^{T} x^{*}+\beta}$

So the curve of objective function for Eq. 1 can be written as

$$
\left(c^{T}-F^{*} d^{T}\right) x=
$$

$\beta F^{*}-\gamma$.

The converted linear programming problem can be obtained

Maximize $\varphi(x)=\left(c^{T}-F^{*} d^{T}\right) x$

Subject to,

$x \in X=\{x: A x \leq b\}, x \geq 0$.

\section{Proposition (1)}

If $x^{*}$ solves Eq. 1 with objective function values $F^{*}$ then $x^{*}$ solves the problem in Eq. 3 with objective value $\varphi^{*}=\beta F^{*}-\gamma$.

Now the problem in Eq. 3 can be rewritten in the form

Maximize $Z=C^{T} X$

Subject to,

$x \in X=\{x: A x \leq b\}$ where, $C^{T}$ is a vector represented by $\left(c^{T}-F^{*} d^{T}\right) \quad$ and $\quad C, x \in \mathbb{R}^{n}$, $A$ is a $n(m+n)$ matrix, $b \in \mathbb{R}^{n}$.

Note that the nonnegative conditions are in the set of constraints.

\section{Solution of Linear Fractional Programming Problems Based on New Ranking Function.}

The proposed new technique for solving the (FLFP) problem can be summarized by the following steps:

Step1: Consider the general form of our problem:

Maximize

$$
F(X)=\frac{c^{T} X+\gamma}{d^{T} X+\beta}
$$

With the constraints $\quad x \in X=$ $\{x: A x \leq b\}, x \geq 0$.

Step2: Convert the problem in Eq. 4 to linear programming problem.

Step 3: Select a feasible point $x^{*}$ and by using Eq. 2 to calculate $F^{*}$.

Step 4: Find the level curve of objective function

$\left(c^{T}-F^{*} d^{T}\right) x=\beta F^{*}-\gamma$.

Step 5: The LPP is transformed into FLPP.

Step 6: Apply the proposed ranking function to get a crisp valued LPP.

Step 7: Solve the obtained crisp value in LPP and the optimal solution can be calculated by using win QSB program (simplex method). 


\section{Numerical Examples}

In this section some examples are tested to illustrate the efficiency of the suggested solution algorithm.

Example1: Let us consider the following linear fractional programming problem

$\operatorname{Max} Z=\frac{x_{2}+1}{x_{1}+3}$

Subject to

$-x_{1}+x_{2} \leq 1$

$$
x_{2} \leq 2
$$

$x_{1}+2 x_{2} \leq 1$

$$
x_{1} \leq 5
$$

$x_{1}, x_{2} \geq 0$.

Let $x^{*}=\left(\begin{array}{l}1 \\ 1\end{array}\right)$, then $F^{*}=\frac{1+1}{1+3}=\frac{1}{2}$ and hence we have

$$
\begin{aligned}
& \left(c^{T}-F^{*} d^{T}\right) x=\left[\begin{array}{ll}
0 & 1
\end{array}\right)-\frac{1}{2}(1 \\
& \left(\begin{array}{l}
x_{1} \\
x_{2}
\end{array}\right)=-\frac{1}{2} x_{1}+x_{2} .
\end{aligned}
$$

Therefore, we have the following LP problem

$\operatorname{Max} Z=-\frac{1}{2} x_{1}+x_{2}$

$$
\begin{aligned}
-x_{1}+x_{2} & \leq 1 \\
x_{2} & \leq 2 \\
x_{1}+2 x_{2} & \leq 1 \\
x_{1} & \leq 5 \\
x_{1}, x_{2} & \geq 0 .
\end{aligned}
$$

By using win QSB program (simplex method) one can get the optimal solution $x_{1}=0, \quad x_{2}=0.5, \quad \operatorname{Max} Z=$ 0.5000 .

Now the LPP is transformed into FLPP.

Let $\Delta_{1}=0.3$ and $\Delta_{2}=1.9$ where $\left(c_{i j}\right.$ $\left.\Delta_{1}, c_{i j}, c_{i j}+\Delta_{2}\right)$ and $i=1,2, j=1,2$.

$\operatorname{Max} Z=-(0.2,0.5,2.4) x_{1}$

$$
+(0.7,1,2.9) x_{2}
$$

Subject to

$$
\begin{aligned}
-x_{1}+x_{2} & \leq(0.7,1,2.9) \\
x_{2} & \leq(1.7,2,3.9) \\
x_{1}+2 x_{2} & \leq(0.7,1,2.9) \\
x_{1} & \leq(4.7,5,6.9) \\
x_{1}, x_{2} & \geq 0 .
\end{aligned}
$$

By applying the ranking function to form the new algorithm in the FLPP and get, a crisp valued LPP. By solving the crisp value in LPP one can get the optimal solution which is listed in Table 1 using different values of $w$. The obtained results by [11] are also given in Table 1 .

\section{Table 1 the optimal solution of}

\section{Example 1}

\begin{tabular}{|c|c|c|c|}
\hline Weight & $\begin{array}{c}\text { Our } \\
\text { results }\end{array}$ & $\begin{array}{c}\text { Results } \\
\text { in [11] }\end{array}$ & $\begin{array}{c}\text { Exact } \\
\text { error }\end{array}$ \\
\hline 0 & 0.8668 & 1.3338 & 0.3668 \\
\hline 0.1 & 0.8192 & 1.2168 & 0.3192 \\
\hline 0.2 & 0.7729 & 1.1050 & 0.2729 \\
\hline 0.3 & 0.7279 & 0.9987 & 0.2279 \\
\hline 0.4 & 0.6844 & 0.8978 & 0.1844 \\
\hline 0.5 & 0.6422 & 0.8021 & 0.1422 \\
\hline 0.6 & 0.6013 & 0.7120 & 0.1013 \\
\hline 0.7 & 0.5618 & 0.6272 & 0.0618 \\
\hline 0.8 & 0.5236 & 0.5477 & 0.0236 \\
\hline 0.9 & 0.4867 & 0.4737 & 0.0133 \\
\hline 1 & 0.4512 & 0.4050 & 0.0488 \\
\hline
\end{tabular}


Example 2: Let us consider the following linear fractional programming problem

$\operatorname{Max} Z=\frac{5 x_{1}+3 x_{2}}{5 x_{1}+2 x_{2}+1}$

Subject to

$3 x_{1}+5 x_{2} \leq 15$

$5 x_{1}+2 x_{2} \leq 10$

$x_{1}, x_{2} \geq 0$

Let $x^{*}=\left(\begin{array}{l}1 \\ 1\end{array}\right)$, then $F^{*}=\frac{5+3}{5+2+1}=1$ and hence we have

$\left(\begin{array}{lll}c^{T} & - & F^{*} d^{T}\end{array}\right) \quad x=\left[\left(\begin{array}{ll}5 & 3\end{array}\right)-\right.$ $\left.1\left(\begin{array}{ll}5 & 2\end{array}\right)\right]\left(\begin{array}{l}x_{1} \\ x_{2}\end{array}\right)=x_{2}$.

Therefore, we have the following LP problem

$\operatorname{Max} Z=x_{2}$

Subject to

$3 x_{1}+5 x_{2} \leq 15$

$5 x_{1}+2 x_{2} \leq 10$

$x_{1}, x_{2} \geq 0$.

We get the optimal solution $x_{1}=0$, $x_{2}=3, \operatorname{Max} Z=3.000$.

Now the LPP is transformed into FLPP.

Let $\Delta_{1}=0.8$ and $\Delta_{2}=1.4$ where $\left(c_{i j}\right.$ $\left.\Delta_{1}, c_{i j}, c_{i j}+\Delta_{2}\right)$ and $i=1,2, j=1,2$.

$\operatorname{Max} Z=(0.2,1,2.4) x_{2}$

Subject to

$3 x_{1}+5 x_{2} \leq(14.2,15,16.2)$
$5 x_{1}+2 x_{2} \leq(9.2,10,11.4)$

$x_{1}, x_{2} \geq 0$.

By applying the ranking function to form the new algorithm in the FLPP and get, a crisp valued LPP. By solving the crisp value in LPP, one can get the optimal solution which is listed in Table 2 using different values of $w$. The obtained results by [11] are also given in Table 2 .

\section{Table 2 the optimal solution of} Example 2.

\begin{tabular}{|c|c|c|c|}
\hline Weight & $\begin{array}{c}\text { Our } \\
\text { results }\end{array}$ & $\begin{array}{c}\text { Results } \\
\text { in [11] }\end{array}$ & $\begin{array}{c}\text { Exact } \\
\text { error }\end{array}$ \\
\hline 0 & 3.7574 & 15.0298 & 0.7574 \\
\hline 0.1 & 3.6369 & 14.5481 & 0.6369 \\
\hline 0.2 & 3.5171 & 14.0685 & 0.5171 \\
\hline 0.3 & 3.3976 & 13.5904 & 0.3976 \\
\hline 0.4 & 3.2786 & 13.1151 & 0.2786 \\
\hline 0.5 & 3.1605 & 12.6420 & 0.1605 \\
\hline 0.6 & 3.0426 & 12.1704 & 0.0426 \\
\hline 0.7 & 2.9252 & 11.7016 & 0.0748 \\
\hline 0.8 & 2.8087 & 11.2349 & 0.1913 \\
\hline 0.9 & 2.6924 & 10.7697 & 0.3076 \\
\hline 1 & 2.5767 & 10.3055 & 0.4233 \\
\hline
\end{tabular}

Example 3: Let us consider the following linear fractional programming problem

$\operatorname{Max} Z=\frac{x_{1}+2 x_{2}}{2 x_{1}-x_{2}+2}$

Subject to

$-x_{1}+2 x_{2} \leq 2$

$$
x_{1}+x_{2} \leq 4
$$

$x_{1}, x_{2} \geq 0$.

Let $x^{*}=\left(\begin{array}{l}1 \\ 1\end{array}\right)$, then $F^{*}=\frac{1+2}{2-1+2}=1$ and hence we have 


$$
\begin{aligned}
& \left(\begin{array}{lll}
c^{T} & - & F^{*} d^{T}
\end{array}\right) \quad x=\left[\left(\begin{array}{ll}
1 & 2
\end{array}\right)-\right. \\
& \left.1\left(\begin{array}{ll}
2 & -1
\end{array}\right)\right]\left(\begin{array}{l}
x_{1} \\
x_{2}
\end{array}\right)=-x_{1}+3 x_{2} \text {. }
\end{aligned}
$$

Therefore, we have the following LP problem

$\operatorname{Max} Z=-x_{1}+3 x_{2}$

Subject to

$$
\begin{aligned}
-x_{1}+2 x_{2} & \leq 2 \\
x_{1}+x_{2} & \leq 4 \\
x_{1}, x_{2} & \geq 0 .
\end{aligned}
$$

We get the optimal solution $x_{1}=$ $2.0000, \quad x_{2}=2.0000, \quad \operatorname{Max} Z=$ 4.000 .

Now the LPP is transformed into FLPP. Let $\Delta_{1}=0.1$ and $\Delta_{2}=1.3$ where $\left(c_{i j}\right.$ $\left.\Delta_{1}, c_{i j}, c_{i j}+\Delta_{2}\right)$ and $i=1,2, j=1,2$.

$\operatorname{Max} Z=-(0.9,1,2.3) x_{1}+$ $(2.9,3,4.3) x_{2}$

Subject to

$$
\begin{aligned}
& -x_{1}+2 x_{2} \leq(1.9,2,3.3) \\
& x_{1}+x_{2} \leq(3.9,4,5.3) \\
& x_{1}, x_{2} \geq 0 .
\end{aligned}
$$

By applying the ranking function to form the new algorithm in the FLPP and get, a crisp valued LPP. By solving the crisp value in LPP, one can get the optimal solution, which is listed, in Table 3 using different values of $w$. The obtained results by [11] are also given in Table 3 .
Table 3 the optimal solution of Example 3.

\begin{tabular}{|c|c|c|c|}
\hline Weight & $\begin{array}{c}\text { Our } \\
\text { results }\end{array}$ & $\begin{array}{c}\text { Results } \\
\text { in [11] }\end{array}$ & $\begin{array}{c}\text { Exact } \\
\text { error }\end{array}$ \\
\hline 0 & 4.3766 & 4.7847 & 0.3766 \\
\hline 0.1 & 4.3346 & 4.6942 & 0.3346 \\
\hline 0.2 & 4.2930 & 4.6052 & 0.2930 \\
\hline 0.3 & 4.2515 & 4.5175 & 0.2515 \\
\hline 0.4 & 4.2106 & 4.4313 & 0.2106 \\
\hline 0.5 & 4.1700 & 4.3467 & 0.1700 \\
\hline 0.6 & 4.1296 & 4.2633 & 0.1296 \\
\hline 0.7 & 4.0898 & 4.1815 & 0.0898 \\
\hline 0.8 & 4.0503 & 4.1804 & 0.0503 \\
\hline 0.9 & 4.0110 & 4.0222 & 0.0110 \\
\hline 1 & 3.9723 & 3.9447 & 0.0277 \\
\hline
\end{tabular}

\section{Conclusion}

We have solved the linear fractional programming problem using a new ranking method which is very easy for computation. A comparative study has been made by considering three examples using the proposed new ranking method. The examples are given to demonstrate the proposed solution.

\section{References}

[1] Alkanani I H and Adnan F A., Ranking Function Methods for Solving Fuzzy Linear Programming Problems, Mathematical Theory and Modeling ,Vol. 4, No. 4, pp. 65-72, (2014).

[2] Allahviranloo T, Lotfi F H, Kiasary M Kh, Kiani N A, Alizadeh L., Solving Fully Fuzzy Linear Programming Problem by the Ranking Function, Applied Mathematical Sciences ,Vol. 1, No. 19, pp.19 - 32, (2008). 
[3] AnithaKumari T, Venkateswarlu B., Murthy A S. Fuzzy Transportation Problems with New Kind of Ranking Function. The International Journal of Engineering and Science, Vol. 6, No.11, pp. 2319 - 1805, (2017).

[4] Chandrasekaran S, Gokila G and Saju J., ranking of octagonal fuzzy numbers for solving multi objective fuzzy linear programming problem with simplex method and graphical method, International journal of scientific engineering and applied science, Vol. 1, No. 5, pp. 2395-3470, (2015).

[5] Das S K and Mandal T., A new model for solving fuzzy linear fractional programming problem with ranking function, Journal of Applied Research on Industrial Engineering, Vol. 4, No. 2, pp. 89-96, (2017).

[6] Dinagar D S, Jeyavuthin M M., Fully Fuzzy Integer Linear Programming Problems Under Robust Ranking Techniques, International Journal of Mathematics and its Applications, Vol. 6, No. 3, pp.19-25, (2018).

[7] Dinagar D S..and Kamalanathan S., Solving Fuzzy Linear Programming Problem Using New Ranking Procedures of Fuzzy Numbers, International Journal of Applications of Fuzzy Sets and Artificial Intelligence, Vol. 7, pp. 281292, (2017).

[8] Ganesh A H and Shobana A H., On Fuzzy Critical Path Method Based On Ranking Of Various Type-2 Fuzzy Quantities Using Centroid Of Centroids, International Journal of Engineering and Advanced Technology, Vol. 8, No. 6, pp. 910-920, (2019).

[9] Gani A N and Mohamed V N., A Method of Ranking Generalized Trapezoidal Intuitionistic Fuzzy Numbers, International Journal of Applied Engineering Research, Vol. 10, No.10, pp. 25465-25473, (2015).
[10] Gong Z, Zhao W and Liu K., A STRAIGHTFORWARD APPROACH FOR SOLVING FULLY FUZZY LINEAR PROGRAMMING PROBLEM WITH LR-TYPE FUZZY NUMBERS, Journal of the Operations Research Society of Japan, Vol. 61, No. 2, pp. 172-185, (2018).

[11] Hasan I, Rahim A, Hasan A., solving fuzzy transportation problem by using ranking function with triangular membership. Baghdad, University of Baghdad College of Science for women department of mathematics, (2015).

[12] Krishnaveni G and Ganesan K., A new approach for the solution of fuzzy games.National Conference on Mathematical Techniques and its Applications, Vol. 18, pp. 1-6, (2018).

[13] Kumar M R, Subramanian S., Solution of Fuzzy Transportation Problems with Triangular Fuzzy Numbers using Ranking Function, International Journal of Pure and Applied Mathematics, Vol. 119, No. 15, pp. 2669-2682, (2018).

[14] Mitlif R J., Solving fuzzy fractional linear programming problems by ranking function methods, JOURNAL OF COLLEGE OF EDUCATION, Vol. 1, pp. 93-108, (2016).

[15] Mitlif R J., A New Method for Solving Fully Fuzzy Multi-Objective Linear Programming Problems, Iraqi Journal of Science, Vol.57(3C), pp. 2307-2311, (2016).

[16] Muralidaran C and Venkateswarlu B., Accuracy Ranking Function for Solving Hexagonal Fuzzy Linear Programming Problem, International Journal of Pure and Applied Mathematics, Vol. 115, No.9, pp.215222, (2017).

[17] Nehi H M., Alineghad M. Solving Interval and Fuzzy Multi Objective Linear Programming Problem by 
Necessarily Efficiency Points, International Mathematical Forum, Vol. 3, No.3, pp. 99 - 106, (2008).

[18] Pandian P., Multi-objective Programming Approach for Fuzzy Linear Programming Problems. Applied Mathematical Sciences, Vol. 7, No. 37, pp. 1811 - 1817, (2013).

[19] Ponnivalavan K and Pathinathan T., Ranking of a Pentagonal Fuzzy Number and Its Applications., Journal of Computer and Mathematical Sciences, Vol. 6, No. 11, pp. 571-584, (2015).

[20] Seikh M R, Nayak P K and Pal M., Solving Bi-matrix Games with Pay-offs of Triangular, Intuitionistic Fuzzy Numbers. EUROPEAN JOURNAL OF PURE AND APPLIED MATHEMATICS, Vol. 8, No. 2, pp.153-171, (2015).

[21] Simi F A and Talukder M S., A New Approach for solving linear fractional programming problems with duality concept, scientific research publishing, Vol. 6, No. 1, pp. 1-10, (2017).

[22] Sudha A S, Vimalavirginmary S and Sathya S., A Novel Approach for Solving Fuzzy Linear programming Problem using Pentagonal Fuzzy numbers, International Journal of Advanced Research, Vol. 4, No. 1, pp. 42-45, (2017). 